\title{
SOME UNUSUAL GENERATING INTEGRALS INVOLVING HERMITE POLYNOMIALS
}

\author{
Ahmed. M. Youssef \\ Basic Science Department, Banha Faculty of Engineering, Banha University, Banha, Egypt. \\ Corresponding author: Ahmed-yousef201480@yahoo.com
}

\section{ABSTRACT}

In this paper we seek to find some bilateral generating functions for some orthogonal polynomials like Hermite, Charlier, Modified Laguerre and Gegenbaur polynomials. These bilateral generating functions can be obtained with the help of the unusual integral formula for Hermite polynomial seen in equation (1.1).

Keywords: Hermite polynomials, Modified Laguerre, Gegenbaur polynomials

\section{INTRODUCTION}

In a recent paper [1] the author established the following unusual integral for Hermite polynomials in the form

$$
\mathrm{H}_{\mathrm{n}}(\mathrm{x})=\frac{1}{\sqrt{\pi}} \int_{-\infty}^{\infty} \exp \left(-\mathrm{v}^{2}\right)[2(\mathrm{x}+\mathrm{iv})]^{\mathrm{n}} d v
$$

The aim of this paper is to find some unusual bilinear generating integrals by the help of the above equation (1.1).

Now, we can find the following bilinear generating functions of Hermite polynomials by using the unusual generating integral (1.1) as follows

$$
\begin{aligned}
\sum_{\mathrm{n}=0}^{\infty} \frac{\mathrm{t}^{\mathrm{k}}}{2^{\mathrm{k}} \mathrm{k} !} \mathrm{H}_{\mathrm{k}}(x) & \mathrm{H}_{\mathrm{k}}(\mathrm{y}) \\
& =\frac{1}{\sqrt{\pi}} \int_{-\infty}^{\infty} \exp \left(-\mathrm{v}^{2}+2 \mathrm{xt}(\mathrm{y}+\mathrm{iv})-\mathrm{t}^{2}\left(\mathrm{y}+\mathrm{iv} \mathrm{v}^{2}\right)\right) \mathrm{dv}
\end{aligned}
$$

The above bilinear generating relation (1.2) is deduced by Chatterjea S. K [1].

\section{Bilateral Generating Function With Charlier and Hermite Polynomials}

Here we notice the following linear generating relation involving Charlier polynomials [2]

$$
\sum_{\mathrm{n}=0}^{\infty} \mathrm{C}_{\mathrm{n}}(k ; \alpha) \frac{t^{\mathrm{n}}}{\mathrm{n} !}=\exp (\mathrm{t})\left(1-\frac{\mathrm{t}}{\alpha}\right)^{\mathrm{k}}
$$

Now, we have 


$$
\begin{gathered}
\sum_{\mathrm{n}=0}^{\infty} \frac{\mathrm{t}^{\mathrm{n}}}{\mathrm{n} !} \mathrm{C}_{\mathrm{n}}(\mathrm{k} ; \alpha) \mathrm{H}_{\mathrm{n}}(\mathrm{x}) \\
=\frac{1}{\sqrt{\pi}} \int_{-\infty}^{\infty} \exp \left(-\mathrm{v}^{2}\right)\left(\sum_{n=0}^{=\infty} \frac{[2 \mathrm{t}(\mathrm{x}+\mathrm{iv})]^{\mathrm{n}}}{\mathrm{n} !} \mathrm{C}_{\mathrm{n}}(k ; \alpha) d v\right. \\
=\frac{1}{\sqrt{\pi}} \int_{-\infty}^{=\infty} \exp \left(-\mathrm{v}^{2}\right) \exp (2 \mathrm{t}(\mathrm{x}+\mathrm{iv}))\left(1-\frac{2 \mathrm{t}(\mathrm{x}+\mathrm{iv})}{\alpha}\right)^{k} \mathrm{dv} \\
=\exp (2 \mathrm{xt})\left(\frac{\mathrm{t}}{\alpha}\right)^{\mathrm{k}} \frac{1}{\sqrt{\pi}} \int_{-\infty}^{\infty} \exp \left(-\mathrm{v}^{2}+2 \mathrm{ivt}\right)\left(\frac{\alpha}{\mathrm{t}}-2(\mathrm{x}+\mathrm{iv})\right)^{\mathrm{k}} d \mathrm{v}
\end{gathered}
$$

Now in order to evaluate (2.2) we shall prove the following integral formula

$$
\frac{1}{\sqrt{\pi}} \int_{-\infty}^{\infty} \exp \left(-\mathrm{v}^{2}+2 \mathrm{ivt}\right)(2(\mathrm{x}+\mathrm{iv}))^{\mathrm{k}} \mathrm{dv}=\exp \left(-\mathrm{t}^{2}\right) \mathrm{H}_{\mathrm{k}}(\mathrm{x}-\mathrm{t})
$$

In fact we notice that

$$
\sum_{\mathrm{n}=0}^{\infty} \mathrm{H}_{\mathrm{n}+\mathrm{k}}(x) \frac{t^{\mathrm{n}}}{\mathrm{n} !}=\exp \left(2 \mathrm{xt}-\mathrm{t}^{2}\right) H_{k}(\mathrm{x}-\mathrm{t})
$$

Now the left member of (2.4) can be written by virtue of (1.2) as follows

$$
\begin{aligned}
\frac{1}{\sqrt{\pi}} \sum_{\mathrm{n}=0}^{\infty} \frac{\mathrm{t}^{\mathrm{n}}}{\mathrm{n} !} \int_{-\infty}^{\infty} \exp & \left(-\mathrm{v}^{2}\right)[2(\mathrm{x}+\mathrm{iv})]^{\mathrm{n}+\mathrm{k}} d v \\
& =\frac{1}{\sqrt{\pi}} \int_{-\infty}^{\infty} \exp \left(-\mathrm{v}^{2}\right) \exp (2 \mathrm{t}(\mathrm{x}+\mathrm{iv}))[2(\mathrm{x}+\mathrm{iv})]^{\mathrm{k}} \mathrm{dv} \\
& =\exp (2 \mathrm{xt}) \frac{1}{\sqrt{\pi}} \int_{-\infty}^{\infty} \exp \left(-\mathrm{v}^{2}+2 \mathrm{ivt}\right)[2(\mathrm{x}+\mathrm{iv})]^{\mathrm{k}} \mathrm{dv}
\end{aligned}
$$

Comparing the above integral with the right member of (2.4) we obtain (2.3).

Now we are in apposition to evaluate (2.2).

To this end we can alternatively write (2.3) in the form

$$
\frac{1}{\sqrt{\pi}} \int_{-\infty}^{\infty} \exp \left(-\mathrm{v}^{2}+2 \mathrm{ivt}\right)(2(\mathrm{x}-\mathrm{iv}))^{\mathrm{k}} \mathrm{dv}=\exp \left(-\mathrm{t}^{2}\right) \mathrm{H}_{\mathrm{k}}(\mathrm{x}+\boldsymbol{t})
$$

Thus it follows from (2.2) and (2.5) that 


$$
\sum_{\mathrm{n}=0}^{\infty} \mathrm{C}_{\mathrm{n}\left(k_{s} \alpha\right)} \mathrm{H}_{\mathrm{n}}(\mathrm{X}) \frac{\mathrm{t} \mathrm{n}}{\mathrm{n} !}=\left(\frac{\mathrm{t}}{\alpha}\right)^{k} \exp \left(2 \mathrm{xt}-\mathrm{t}^{2}\right) \mathrm{H}_{\mathrm{k}}\left(\frac{2 t^{2}-2 x \mathrm{t}+\alpha}{2 \mathrm{t}}\right)
$$

\section{Bilateral Generating Function with modified Laguerre and Hermite Polynomials}

Here we notice the following linear generating relation involving modified Laguerre polynomials

$$
\sum_{\mathrm{n}=0}^{\infty} \mathrm{L}_{\mathrm{n}}^{\mathrm{k}-\mathrm{n}_{(\alpha)}} \boldsymbol{t}^{n}=\mathrm{e}_{x} \mathrm{p}(-\alpha t)(1+\mathrm{t})^{\mathrm{k}}
$$

Similarly by multiplying the left hand side of the previous equation by $\mathrm{H}_{\mathrm{n}}(\mathrm{x})$ and substitute from (1.2) we get

$$
\begin{aligned}
\sum_{\mathrm{n}=0}^{\infty} \mathrm{L}_{\mathrm{n}}^{\mathrm{k}-\mathrm{n}_{(\alpha)}} & H_{\mathrm{n}}(\mathrm{X}) t^{\mathrm{n}} \\
& =\frac{1}{\sqrt{\pi}} \int_{-\infty}^{\infty} \exp \left(-\mathrm{v}^{2}\right)\left(\sum_{\mathrm{n}=0}^{\infty}[2 \mathrm{t}(\mathrm{x}+\mathrm{iv})]^{\mathrm{n}} \mathrm{L}_{\mathrm{n}}^{\left.\mathrm{k}-\mathrm{n}_{(\alpha)}\right) d v}\right. \\
& =\frac{1}{\sqrt{\pi}} \int_{-\infty}^{\infty} \exp \left(-\mathrm{v}^{2}\right) \exp (-2 \alpha \mathrm{t}(\mathrm{x}+\mathrm{i} v))(1+2 \mathrm{t}(\mathrm{x}+\mathrm{iv}))^{\mathrm{k}} \mathrm{d} v \\
& =\frac{1}{\sqrt{\pi}} \exp (-2 \alpha x \mathrm{t}) \int_{-\infty}^{\infty} \exp \left(-\mathrm{v}^{2}-2 \alpha \operatorname{tiv}\right)(1+2 \mathrm{t}(\mathrm{x}+\mathrm{iv}))^{\mathrm{k}} \mathrm{dv}
\end{aligned}
$$

By replacing $(t)$ by $(-t)$ in the previous equation and substitute from (2.3) we get

$$
\begin{aligned}
& \sum_{\mathrm{n}=0}^{\infty} \mathrm{L}_{\mathrm{n}}^{\mathrm{k}-\mathrm{n}_{(\alpha)}} \quad H_{n}(\mathrm{x})(-\mathrm{t})^{\mathrm{n}} \\
& =\frac{1}{\sqrt{\pi}} \exp (2 \alpha x t) \int_{-\infty}^{\infty} e \mathrm{x} p\left(-\mathrm{v}^{2}+2 \alpha \mathrm{ti} v\right)(1-2 \mathrm{t}(\mathrm{x}+\mathrm{iv}))^{\mathrm{k}} d v \\
& =\frac{1}{\sqrt{\pi}} \exp (2 \alpha x t)(\mathrm{t})^{\mathrm{k}} \int_{-\infty}^{\infty} \exp \left(-\mathrm{v}^{2}+2 \alpha \mathrm{tiv}\right)\left(\frac{1}{\mathrm{t}}-2(\mathrm{x}+\mathrm{iv})\right)^{\mathrm{k}} d \mathrm{~V} \\
& =\frac{1}{\sqrt{\pi}} \exp (2 \alpha x \mathrm{t})(\mathrm{t})^{\mathrm{k}} \int_{-\infty}^{\infty} \exp \left(-\mathrm{v}^{2}+2 \alpha \operatorname{tiv}\right)\left(2\left(\frac{1}{2 \mathrm{t}}-\mathrm{x}-\mathrm{iv}\right)\right)^{\mathrm{k}} \mathrm{d} v
\end{aligned}
$$

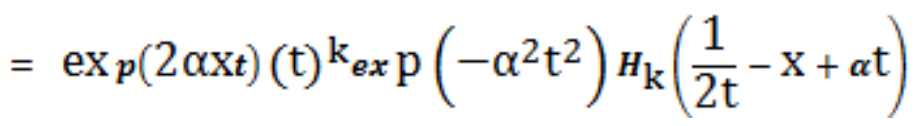

$$
\begin{aligned}
& =\mathrm{t}^{\mathrm{k}} \exp \left(2 \alpha x \mathrm{t}-\alpha^{2} \mathrm{t}^{2}\right) H_{\mathrm{k}}\left(\frac{2 \alpha \mathrm{t}^{2}-2 \mathrm{xt}+1}{2 \mathrm{t}}\right)
\end{aligned}
$$


Hence;

$$
\begin{aligned}
& \sum_{\mathrm{n}=0}^{\infty} \mathrm{L}_{\mathrm{n}}^{\mathrm{k}-\mathrm{n}_{(}}(\alpha) \mathrm{H}_{\mathrm{n}}(\mathrm{x})(-\mathrm{t})^{\mathrm{n}} \\
& \quad=\mathrm{t}^{\mathrm{k}} \exp \left(2 \alpha \mathrm{xt}-\alpha^{2} \mathrm{t}^{2}\right) \mathrm{H}_{\mathrm{k}}\left(\frac{2 \alpha \mathrm{t}^{2}-2 \mathrm{xt}+1}{2 \mathrm{t}}\right)
\end{aligned}
$$

It may be of interest to note that we can obtain equation (3.2) from equation (2.5) so we have to prove that:

$$
\mathrm{C}_{\mathrm{n}}(\mathrm{k}, \alpha)=(-\alpha)^{-\mathrm{n}}{ }_{n} ! \mathrm{L}_{\mathrm{n}}^{\mathrm{k}-\mathrm{n}}(\alpha)
$$

From Laguerre polynomial $\mathrm{L}_{\mathrm{n}}^{\mathrm{X}}(\alpha)$

$$
\mathrm{L}_{\mathrm{n}}(\alpha)=\sum_{\mathrm{r}=0}^{\mathrm{n}} \frac{(-1)^{\mathrm{r}}(1+\mathrm{x})_{\mathrm{n}}}{\mathrm{r} !(\mathrm{n}-\mathrm{r}) !(1+\mathrm{x})_{\mathrm{r}}} \alpha^{\mathrm{r}}
$$

Let $\mathrm{X}=\mathrm{K}-\boldsymbol{n}$, then

$$
\begin{aligned}
\mathrm{L}_{\mathrm{n}}^{\mathrm{k}-\mathrm{n}_{(\alpha)}} & =\sum_{\mathrm{r}=0}^{\mathrm{n}} \frac{(-1)^{\mathrm{r}}(1+\mathrm{k}-\mathrm{n})_{\mathrm{n}}}{\mathrm{r} !(\mathrm{n}-\mathrm{r}) !(1+\mathrm{k}-\mathrm{n})_{\mathrm{r}}} \alpha^{\mathrm{r}} \\
& =\sum_{\mathrm{r}=0}^{\mathrm{n}} \frac{(-1)^{\mathrm{r}}(1+\mathrm{k}-\mathrm{n})_{\mathrm{n}}}{\mathrm{r} !(\mathrm{n}-\mathrm{r}) !(1+\mathrm{k}-\mathrm{n})_{\mathrm{r}}} \alpha^{\mathrm{r}} \\
& =\sum_{\mathrm{r}=0}^{\mathrm{n}} \frac{(-1)^{\mathrm{r}}(-\mathrm{k})_{\mathrm{n}-\mathrm{r}}(-1)^{\mathrm{n}-\mathrm{r}}}{\mathrm{r} !(\mathrm{n}-\mathrm{r}) !} \alpha^{\mathrm{r}} \\
& =\sum_{\mathrm{r}=0}^{\mathrm{n}} \frac{(-1)^{\mathrm{n}}(-\mathrm{k})_{\mathrm{n}-\mathrm{r}}}{\mathrm{r} !(\mathrm{n}-\mathrm{r}) !} \alpha^{\mathrm{r}}
\end{aligned}
$$

Multiplying both sides of the previous equation by $n !(-\alpha)^{-n}$

$$
\begin{aligned}
(-\alpha)^{-\mathrm{n}} n ! \mathrm{L}_{\mathrm{n}}^{\mathrm{k}}-\mathrm{n}(\alpha) & =\sum_{\mathrm{r}=0}^{\mathrm{n}} \frac{(-1)^{\mathrm{n}}(-\mathrm{k})_{\mathrm{n}-\mathrm{r}}}{\mathrm{r} !(\mathrm{n}-\mathrm{r}) !} \alpha^{r}(-\alpha)^{-\mathrm{n}} n ! \\
& =\sum_{\mathrm{r}=0}^{\mathrm{n}} \frac{n !(-\mathrm{k})_{\mathrm{n}-\mathrm{r}}}{\mathrm{r} !(n-r) !} \alpha^{r-\mathrm{n}}
\end{aligned}
$$


Let $\mathrm{m}=\mathrm{n}-\boldsymbol{r}$, hence

$$
\begin{aligned}
& =\sum_{\mathrm{m}=0}^{\mathrm{n}} \frac{\mathrm{n} !(-\mathrm{k})_{\mathrm{m}}}{\mathrm{m} !(\mathrm{n}-\mathrm{m}) !} \alpha^{-\mathrm{m}} \\
& =\sum_{\mathrm{m}=0}^{\mathrm{n}\left(\begin{array}{l}
\mathrm{n} \\
\mathrm{m}
\end{array}\right)(-\mathrm{k})_{\mathrm{m}} \alpha^{-\mathrm{m}}} \\
\text { Since }(-\mathrm{k})_{\mathrm{m}}=(-1)^{\mathrm{m}} & \frac{\mathrm{k} !}{(\mathrm{k}-\mathrm{m} !)} \text { hence; } \\
(-\alpha)^{-\mathrm{n}} n ! \mathrm{L}_{\mathrm{n}}^{\mathrm{k}-\mathrm{n}}(\alpha) & =\sum_{\mathrm{m}=0}^{\mathrm{n}}(-1)^{\mathrm{m}}\left(\begin{array}{l}
\mathrm{n} \\
\mathrm{m}
\end{array}\right) \frac{\mathrm{k} !}{(\mathrm{k}-\mathrm{m} !)} \alpha^{-\mathrm{m}} \\
& =\sum_{\mathrm{m}=0}^{\mathrm{n}}(-1)^{\mathrm{m}}\left(\begin{array}{l}
\mathrm{n} \\
\mathrm{m}
\end{array}\right)\left(\begin{array}{l}
\mathrm{k} \\
\mathrm{m}
\end{array}\right) \frac{\mathrm{m} !}{(\alpha)^{\mathrm{m}}} \\
& =\mathrm{C}_{\mathrm{n}}(\mathrm{k}, \alpha)
\end{aligned}
$$

Hence;

$$
\mathrm{C}_{\mathrm{n}}(\mathrm{k}, \alpha) \frac{\alpha^{\mathrm{n}}}{\mathrm{n} !}=(-1)^{\mathrm{n}} \mathrm{L}_{\mathrm{n}}^{\mathrm{k}-\mathrm{n}_{(\alpha)}}
$$

Finally by substitute from equation (3.4) in equation (2.5) we get

$$
\begin{aligned}
& \sum_{\mathrm{n}=0}^{\infty} \frac{\mathrm{t}}{\mathrm{n}} \frac{\mathrm{n} !}{(-1)^{\mathrm{n}}} \mathrm{L}_{\mathrm{n}}^{\mathrm{k}-\mathrm{n}}(\alpha) \frac{\mathrm{n} !}{\alpha^{\mathrm{n}}} H_{\mathrm{n}}(\mathrm{X}) \\
&=\left(\frac{\mathrm{t}}{\alpha}\right)^{\mathrm{k}} \exp \left(2 \mathrm{xt}-\mathrm{t}^{2}\right) H_{\mathrm{k}}\left(\frac{2 \mathrm{t}^{2}-2 \mathrm{xt}+\alpha}{2 \mathrm{t}}\right)
\end{aligned}
$$

Hence;

$$
\begin{aligned}
\sum_{\mathrm{n}=0}^{\infty}\left(\frac{-\mathrm{t}^{\mathrm{n}}}{\alpha}\right)^{\mathrm{m}} & \mathrm{L}_{\mathrm{n}}^{\mathrm{k}-\mathrm{n}}(\alpha) \mathrm{H}_{\mathrm{n}}(\mathrm{x}) \\
& =\left(\frac{\mathrm{t}}{\alpha}\right) \exp \left(2 \mathrm{xt}-\mathrm{t}^{2}\right) \mathrm{H}_{\mathrm{k}}\left(\frac{2 \mathrm{t}^{2}-2 \mathrm{xt}+\alpha}{2 \mathrm{t}}\right)
\end{aligned}
$$

We notice that we can obtain the same result (3.5) from (3.2) by replacing $(\mathrm{t})$ by $(\mathrm{t} / \alpha)$. 
Here we notice the following linear generating relation involving Gegenbaur polynomials

$$
\sum_{n=0}^{\infty} C_{n}^{v_{n}}(x) t^{n}=\left(1-2 x t+t^{2}\right)^{-v}
$$

Now by multiplying the left hand side of (3.1) by $\mathrm{H}_{\mathrm{n}}(\mathrm{x})$ and substituting by (1.2) we get (3.2)

$$
\begin{aligned}
\sum_{\mathrm{n}=0}^{m} \mathrm{C}_{\mathrm{n}}^{\mathrm{v}}(\mathrm{x}) \mathrm{t}^{\mathrm{n}} \mathrm{H}_{\mathrm{n}}(\mathrm{x}) & \\
= & \frac{1}{\sqrt{\pi}} \int_{-\infty}^{\infty} \exp \left(-\mathrm{v}^{2}\right)\left(\sum_{\mathrm{n}=0}^{\infty}[2 \mathrm{t}(\mathrm{x}+\mathrm{iv})]^{\mathrm{n}} \mathrm{C}_{\mathrm{n}}^{\mathrm{v}}(\mathrm{x})\right) d v \\
& =\frac{1}{\sqrt{\pi}} \int_{-\infty}^{\infty} \exp \left(-\mathrm{v}^{2}\right)\left[1-4 \mathrm{xt}(\mathrm{x}+\mathrm{iv})+(2 \mathrm{t}(\mathrm{x}+\mathrm{iv}))^{2}\right]^{-v} d v
\end{aligned}
$$

In particular case, if we take $\mathrm{X}=1$ in the above equation (4.1), then we have

$$
\begin{aligned}
\sum_{n=0}^{\infty} C_{n}^{v}(1) t^{n} & =\left(1-2 t+t^{2}\right)^{-v} \\
& =(1-t)^{-2 v} \\
& =\sum_{n=0}^{\infty} \frac{(2 v)_{n}}{n !} t^{n}
\end{aligned}
$$

Hence;

$$
\mathrm{C}_{\mathrm{n}}^{\mathrm{v}}(1)=\frac{(2 \mathrm{v})_{\mathrm{n}}}{\mathrm{n} !}
$$

Secondly substitute in (4.2) we get

$$
\begin{aligned}
& \sum_{\mathrm{n}=0}^{\infty} \frac{(2 \mathrm{v})_{\mathrm{n}}}{\mathrm{n} !} H_{\mathrm{n}}(\mathrm{x}) \mathrm{t}^{\mathrm{n}} \\
& \quad=\frac{1}{\sqrt{\pi}} \int_{-\infty}^{\infty} \exp \left(-\mathrm{v}^{2}\right)\left[1-4 \mathrm{t}(\mathrm{x}+\mathrm{iv})+(2 \mathrm{t}(\mathrm{x}+\mathrm{iv}))^{2}\right]^{-\mathrm{v}} \mathrm{dv}
\end{aligned}
$$

Finally we get; 


$$
\begin{aligned}
& \sum_{\mathrm{n}=0}^{\infty}(2 \mathrm{v})_{\mathrm{n}} \mathrm{e}^{2 \mathrm{xt}-\mathrm{t}^{2}} \\
& \quad=\frac{1}{\sqrt{\pi}} \int_{-\infty}^{\infty} \exp \left(-\mathrm{v}^{2}\right)\left[1-4 \mathrm{t}(\mathrm{x}+\mathrm{iv})+(2 \mathrm{t}(\mathrm{x}+\mathrm{iv}))^{2}\right]^{-\mathrm{v}} d \mathrm{v}
\end{aligned}
$$




\section{REFERENCES}

[1] Chatterjea, S. K., My favourite proof of Mehler's formula, to appear in Jour. Pure math. Vol. 2 (1982).

[2] Lee, P. A., Some generating function involving the Charlier's and Hermite polynomials, Nanta. Math. 8 (1975), 83-87.

[3] Rainville, E. D., Special function, New Yourk (1960).

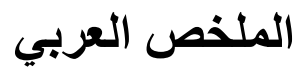

يتتاول هـ ذا البحـث بعض التعـاريف الهامـة بالنسبة الـي كثيـرة حدود هارميـت بالاضـافة الي عمليـة

الاندماج مع بعض كثيرة الحدود الاخري مثال لـ ذلك كثيرة حدود لاجيـر وكثيرة حدود تشـارلز وكذذلك الاندماج مع كثيرة حدود جاجن بيور مما يعطي اضافة للبحث في مجال تعريف الـدوال الخطيـة و الـدوال ثنائي الخطية . 